\title{
Virtual embryoscope for detection of structural abnormalities in first trimester pregnancy
}

\section{Sumesh Choudhary*, Vineet Mishra, Rohina Aggarwal}

Department of Obstetrics and Gynaecology, Institute of Kidney Diseases and Research Centre, Ahmedabad, Gujarat, India

Received: 21 February 2017

Accepted: 27 March 2017

\section{*Correspondence:}

Dr. Sumesh Choudhary,

E-mail: drsumeshchoudhary@gmail.com

Copyright: (C) the author(s), publisher and licensee Medip Academy. This is an open-access article distributed under the terms of the Creative Commons Attribution Non-Commercial License, which permits unrestricted non-commercial use, distribution, and reproduction in any medium, provided the original work is properly cited.

\section{ABSTRACT}

Background: To determine whether there is an additional value of virtual embryoscopy with 3Dimensional (3D) ultrasound above (2D) ultrasound regarding detection rates of structural abnormalities in first trimester pregnancy.

Methods: This was a prospective study consisting of 52 pregnant women with gestational age ranging from 6 to 12 weeks. 2D and 3D ultrasound scan was performed using GE voluson E8 transvaginal probe. All scans were evaluated by ten gynecologists trained in USG. The observations were noted and compared for anatomical details and detection of structural defects.

Results: It was found that images were seen well with 3D (virtual embryoscope). i.e. maximum count 19 in the table and there is statistically significant difference between 3D and 2D images with a p-value $<0.01$ at $95 \%$ confidence interval (CI).

Conclusions: Virtual embryoscope is an innovative tool for evaluation of developing embryo and early foetal morphology. The advantages are the ability to reconstruct $3 \mathrm{D}$ images with the stored scanned volume and examine without having to rescan the patient.

Keywords: 3Dimensional ultrasound, First trimester, Structural abnormalities, Virtual embryoscope

\section{INTRODUCTION}

In the earlier days, first trimester ultrasound was used for confirming fetal viability and determining the gestational age. With the advent of virtual embryoscope, it has now become possible to effectively screen and detect structural abnormalities in first trimester. Threedimensional ultrasound is a useful tool for research in fetal embryology. However, there is no evidence that the results of 3D ultrasound alter the clinical management over standard 2D ultrasound such that outcomes are improved. Whether 3D ultrasound provides unique, clinically relevant information remains to be determined. ${ }^{1}$ The use of 3D ultrasound in the detection of foetal anomalies, especially the anomalies of face, limbs, thorax and spine has been applied by numerous centres across the world. ${ }^{2}$ The use of 3D and 4D ultrasound in early pregnancy assessment has recently been summarized by zanforlin et al. ${ }^{3}$

The aim of the study was to determine whether there is an additional value of virtual embryoscopy with 3D ultrasound above 2D ultrasound in detection of structural abnormalities in the fetus in first trimester.

\section{METHODS}

This was a prospective study conducted in the Department of Obstetrics and Gynecology at Institute of Kidney Disease and Research Centre (IKDRC) from January 2016 to June 2016. The Study population comprised of 52 pregnant women attending the hospital with gestational age ranging from 6 to 12 weeks and consisted of singleton, twin, triplet, quadruplet 
pregnancies and pregnancies with missed abortion. Out of 52 females, 31 conceived spontaneously, 16 after IVF/ICSI (In Vitro Fertilisation/Intra Cytoplasmic Sperm Injection) while 5 after IUI (Intra Uterine Insemination). Gestational age estimation in patients who conceived spontaneously was based on as derived by first day of last menstrual period while for patients who conceived after IUI/IVF/ICSI was based on date of IUI or date of embryo transfer. Informed consent was obtained from all women participating in the study. Transvaginal sonography (TVS) was done using GE Voluson E8 machine. 2D and 3D scans were performed on all the participants. 3D data base was saved and 3D volumes were resized, rotated and cropped when necessary and gray scales and opacity values were adjusted for optimal image quality. All fifty scans were evaluated and analyzed by ten gynecologists trained in ultrasonography (USG) and were coined as NADD (No Anatomical Details detected), Normal, Better and Excellent. The observations were noted for anatomical details and detection of structural defects.

\section{Statistical analysis}

Statistical analysis was done using data analysis software system, Statistical Package for Social Sciences (SPSS) Version 20. 4*3 $\chi^{2}$ - (chi square) test was applied for analysis of the Data. P-value <0.05 was considered statistically significant.

\section{RESULTS}

Mean age of the patients was $30.04 \pm 4.85$ years, mean body mass index (BMI) $22.05 \pm 2.62 \mathrm{Kg} / \mathrm{m}^{2}$ and mean gestational age was $9.05 \pm 2.84$ weeks (Table 1 ).

Table 1: Patient characteristics.

\begin{tabular}{|l|l|}
\hline Variables & Values \\
\hline Age $(\mathrm{yrs})$ & $30.04 \pm 4.85$ \\
\hline BMI $\left(\mathrm{Kg} / \mathrm{m}^{2}\right)$ & $22.05 \pm 2.62$ \\
\hline Mean Gestational Age (weeks) & $9.05 \pm 2.84$ \\
\hline
\end{tabular}

Serial scans of a developing embryo and fetus from 6-12 weeks with structural details as seen on virtual embryoscopy are shown in Figure 1 to Figure 9.

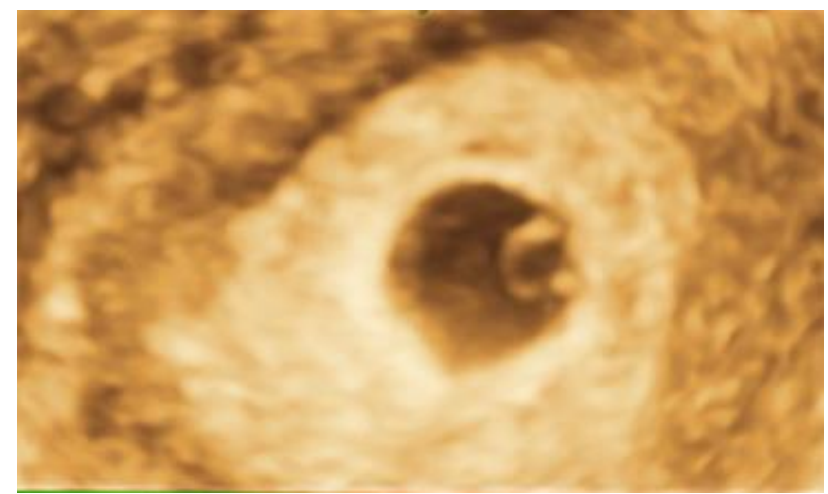

Figure 1: Five-week pregnancy.

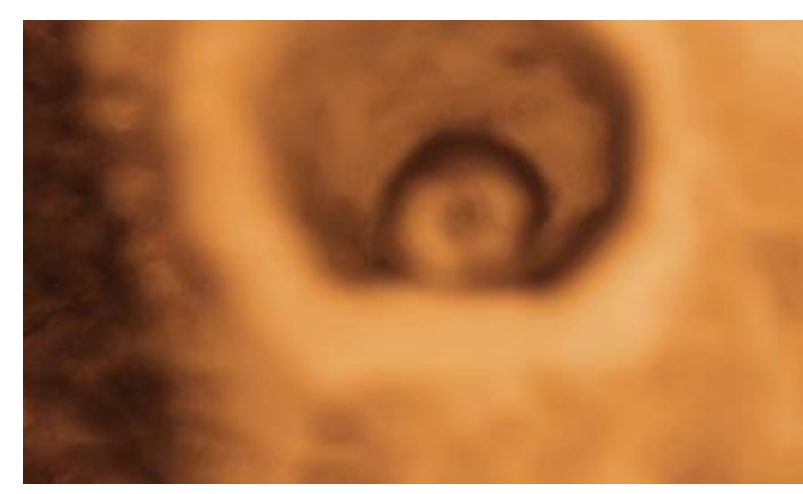

Figure 2: Six-week pregnancy.

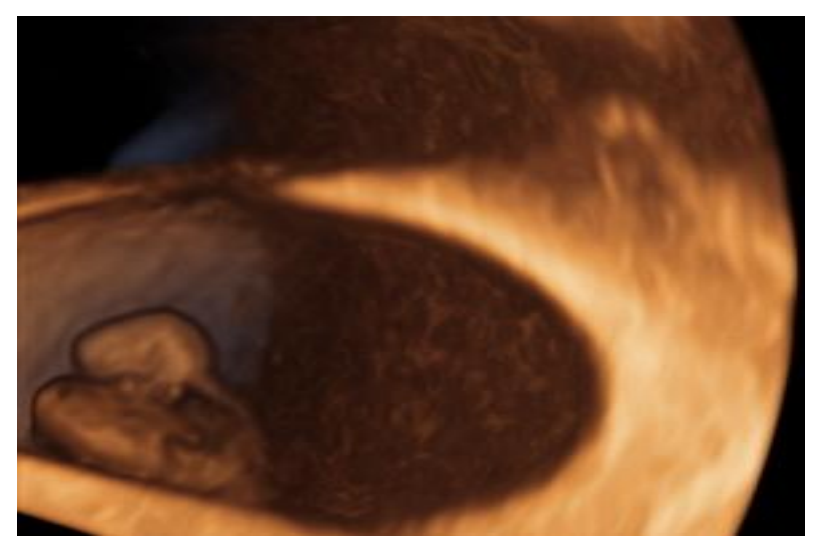

Figure 3: Seven-week pregnancy.

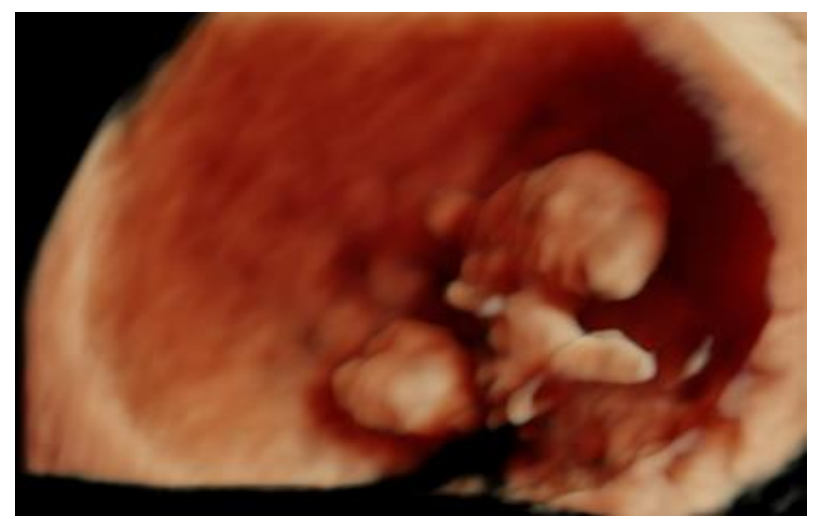

Figure 4: Seven week four days pregnancy.

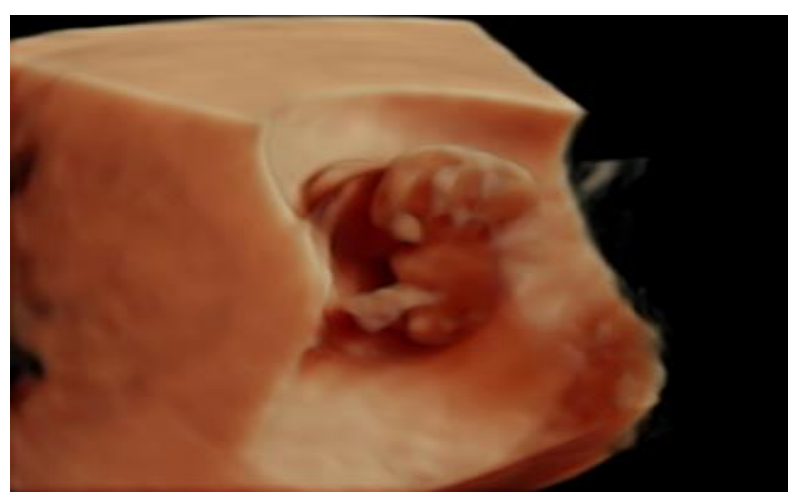

Figure 5: Eight week pregnancy. 


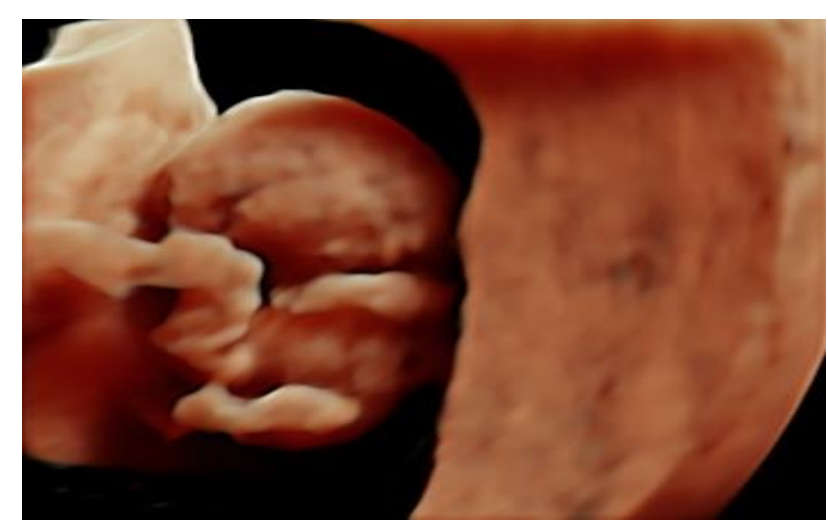

Figure 6: Nine week pregnancy.

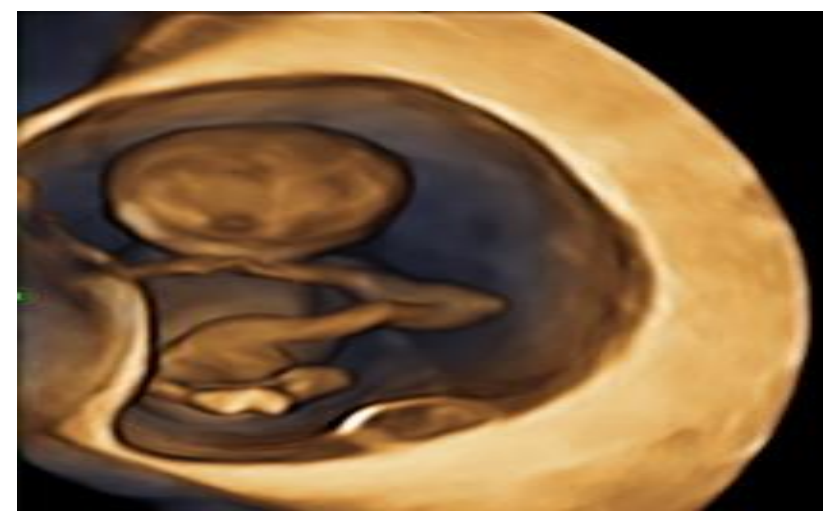

Figure 7: Ten week pregnancy.

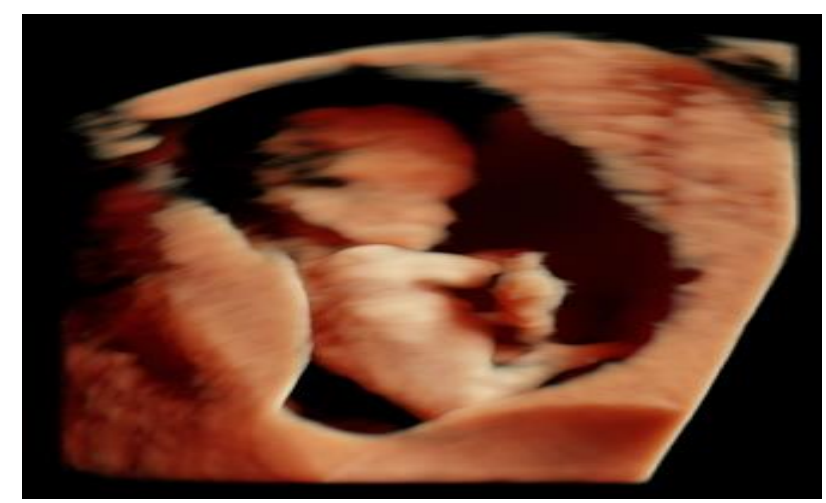

Figure 8: Eleven week pregnancy.

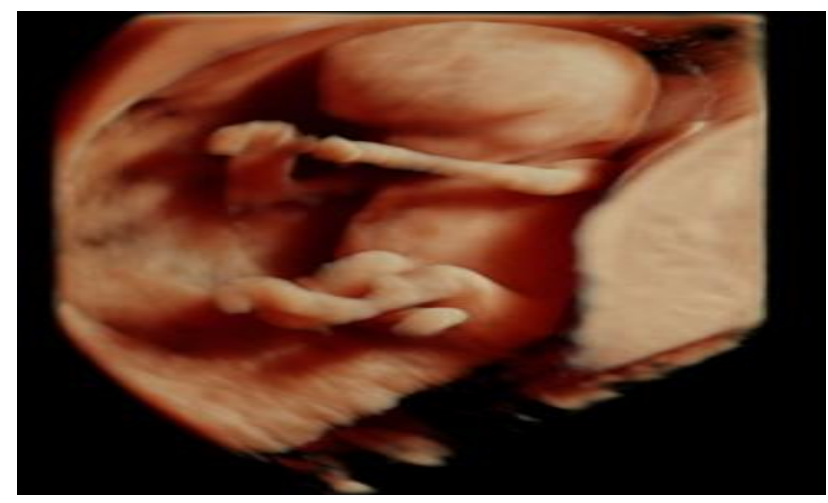

Figure 9: Twelve week pregnancy.
One patient had intrauterine pregnancy with submucous fibroid (Figure 10).

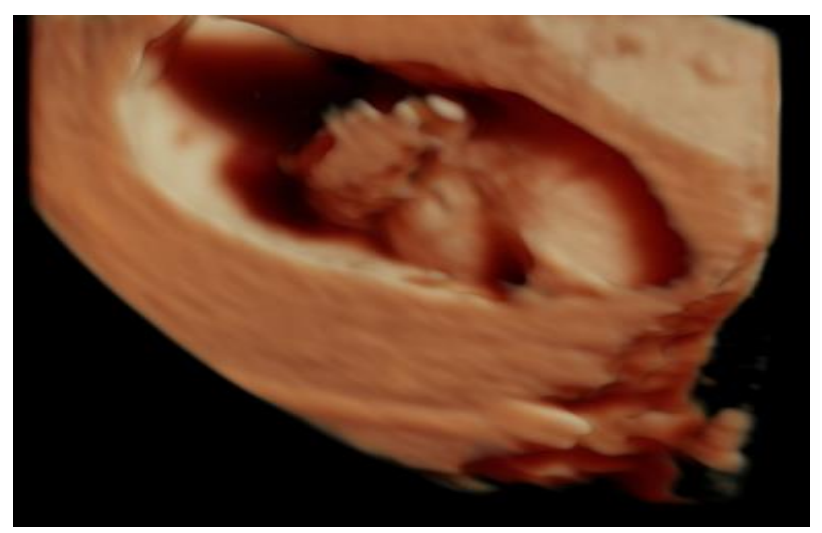

Figure 10: Pregnancy with fibroid.

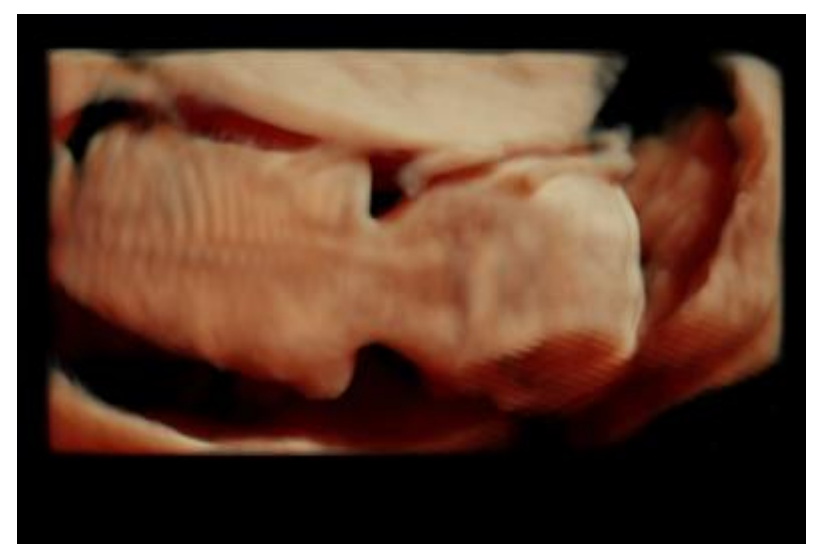

Figure 11: Normal spine twelve week of pregnancy.

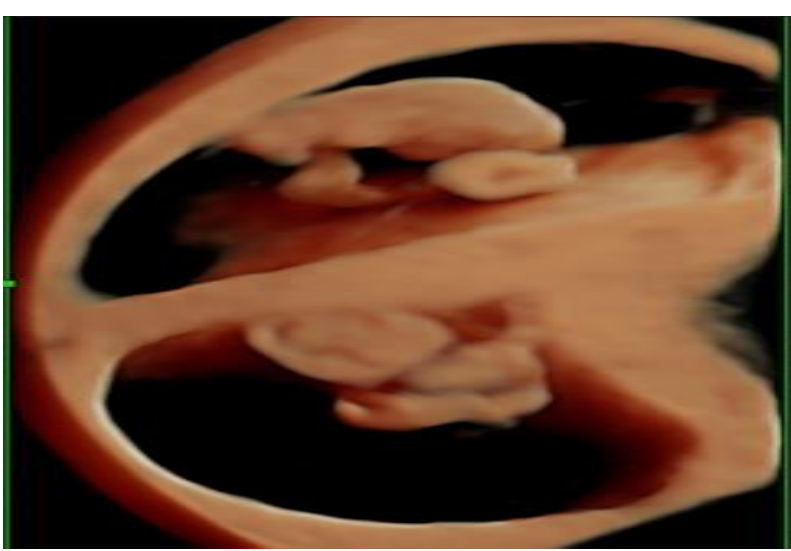

Figure 12: Twin pregnancy.

Figure 11 shows normal fetal spine in a 12-week fetus while multiple pregnancies as seen on virtual embryoscopy are depicted in Figure 12 to Figure 14.

Detailed scan of missed abortion embryo showed membrane separation but no gross structural abnormality was noted (Figure 16). 


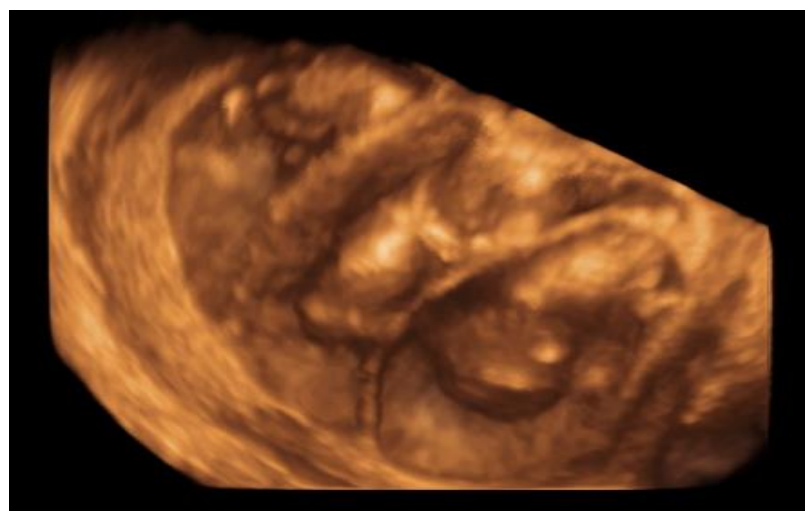

Figure 13: Triplet pregnancy.

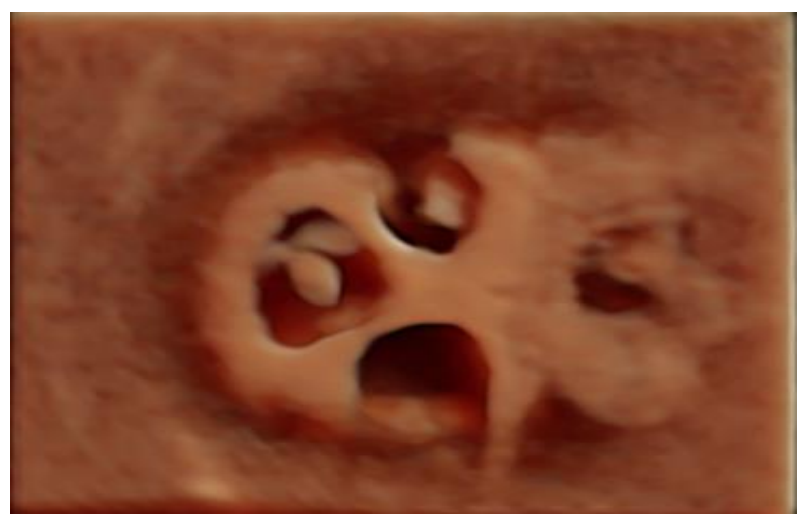

Figure 14: Quariplatepregnancy.

Abnormal nuchal translucency was seen in one fetus (Figure 15).

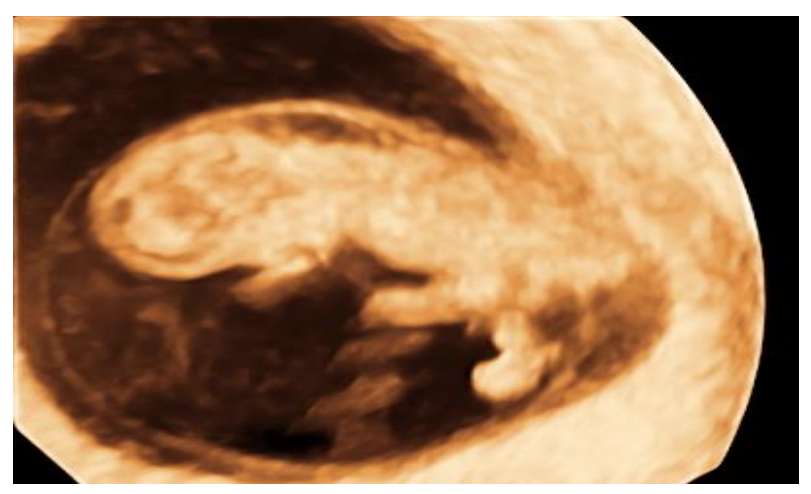

Figure 15: Abnormal nuchal thickness.

Ten Gynecologists evaluated the scans and coined them as NADD (No Anatomical Details detected), Normal, Better and Excellent. Out of 9 scans in which no anatomical details could be seen on 2 D USG, with virtual embryoscopy, it was possible to see normal details in 3 and better anatomical details in 1 but no details could be seen in 5 scans. Out of 39 normal scans on 2D, with 3D we were able to see normal anatomical details in 12 , better details in 19 and excellent in 6 . Out of four images which were seen better on 2D, 2 were also better in 3D while 2 were excellent (Table 2).

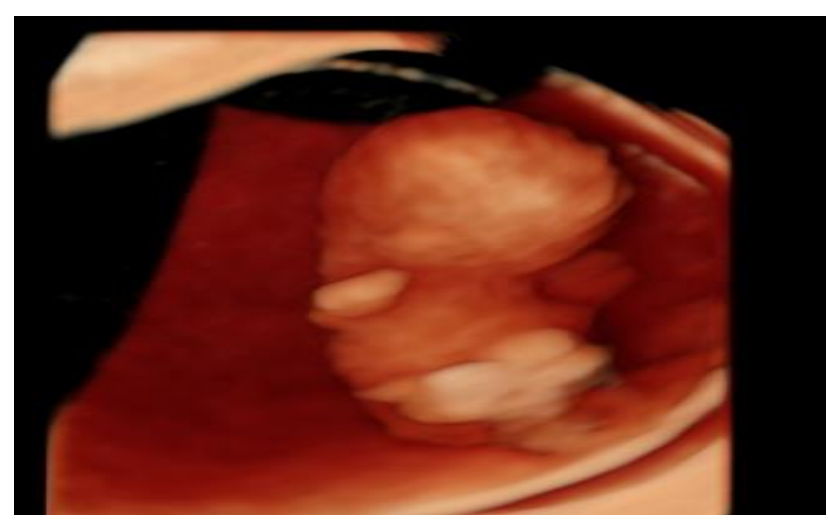

Figure 16: Missed abortion with separation of membrane.

Table 2: Comparison of 2D versus 3D ultrasound in first trimester.

\begin{tabular}{|c|c|c|c|c|c|}
\hline \multirow{2}{*}{ 2D } & \multicolumn{4}{|l|}{ 3D } & \multirow[t]{2}{*}{$P$ value } \\
\hline & NADD & Normal & Better & Excellent & \\
\hline $\begin{array}{l}\text { NADD } \\
(9)\end{array}$ & 4 & 3 & 1 & 0 & \multirow{3}{*}{$<0.01$} \\
\hline $\begin{array}{l}\text { Normal } \\
\text { (39) }\end{array}$ & 2 & 12 & 19 & 6 & \\
\hline $\begin{array}{l}\text { Better } \\
\text { (4) }\end{array}$ & 0 & 0 & 2 & 2 & \\
\hline
\end{tabular}

*(NADD-No Anatomical Details Detected)

So, it was observed that images obtained by 3D (virtual embryoscope) were better than 2D images for first trimester anatomical survey and for detection of structural anomalies i.e. maximum count 19 in table 2 and the difference was statistically significant ( $p$ value $<0.01$ at $95 \% \mathrm{CI})$.

\section{DISCUSSION}

This study demonstrates that it is possible to perform a comprehensive anatomical survey of fetus at 6-12 week gestation using 3D volume in majority of the cases and within a reasonable time frame. Although a complete anatomical survey was frequently completed using realtime 2D scan, the examination of 3D volume irrespective of fetal position provided enough information for comprehensive review of fetal anatomy. ${ }^{4}$

Though 2D ultrasound provided complete anatomical details, the use of 3D volume resulted in reduced scanning time required to obtain this information. The obtained images could be manipulated in order to get all desired views. The potential advantages of virtual embryoscopy in clinical practice could be reduced examination time as well as providing an excellent way to store ultrasound data, allowing the virtual scan to be repeated at any time.

The use of stored 3D volumes has certain limitations including the inability to examine the moving heart. In 
this study, the main factors responsible for failure to complete the anatomical survey were fetal movements during volume acquisition and limited resolution in certain fetal positions. Fetal movements during scan were responsible for failure to demonstrate fetal extremities. Although gross fetal movements could easily be seen during volume acquisition, small movements of the limbs were sometimes not recognized resulting in $3 \mathrm{D}$ volume acquisition artifact. Reduced resolution of structures far away from the vaginal probe limited the advantages of 3D manipulation of obtained volume. The 3D reconstructed view could be as good as the respective $2 \mathrm{D}$ images but the fetal part could not be fully examined in a given volume. ${ }^{5}$

In 1990, Timor- Tritsch et al introduced 'sonoembryology'. They used a high frequency vaginal probe to image early pregnancies. For limb development, a cephalocaudal maturity was described; the tail section protrudes caudally and exceeds the lower limbs. At 7-8 weeks of gestational age, the sole of the feet were found to face each other, and at approximately 10 weeks, rotated ventrally and the legs crossed. ${ }^{2}$ Our results are in accordance with those of Timor-Tritsch et al, and confirm our previous findings that these developing processes can be very well described according to the Carnegie staging system.

In this study we demonstrated the use of an innovative tool for the evaluation of developmental embryonic and early fetal morphology. Combining this measurement with a description of morphological features, such as limb development provides a more solid comprehension of the developing embryo. It can be expected that applying virtual embryoscope will enable us to diagnose growth and /or developmental delay earlier and more accurately. ${ }^{6}$ This is especially important for pregnancies at risk for severe complications such as recurrent late miscarriage, early growth restriction and first trimester detection of structural anomalies like limb defects. Hence, combining length measurements with viewing developmental features using virtual reality techniques, will greatly improve knowledge of normal and abnormal embryonic growth, development and morphology. ${ }^{7}$

In this study, we could not diagnose any significant structural abnormality in 6-9 weeks of pregnancy. The study demonstrates that it is possible to perform comprehensive anatomical survey of fetus at 10-12 weeks using virtual embryoscope. Detailed screening of missed abortion embryo was done but no specific structural abnormality was detected. The limitation of our study was a relatively small sample size and longitudinal data instead of cross -sectional data.
Virtual Embryoscopy opens the way for studying the relationship between embryonic growth, development and morphology as well as second and third trimester pregnancy complications.

\section{CONCLUSION}

Virtual embryoscope is an innovative tool for evaluation of developing embryo and early foetal morphology. The proposed advantages are the ability to reconstruct $3 \mathrm{D}$ views with the stored scanned volume and examine without having to rescan the patient.

Funding: No funding sources

Conflict of interest: None declared

Ethical approval: The study was approved by the Institutional Ethics Committee

\section{REFERENCES}

1. Jurkovi D. Three-dimensional ultrasound in gynecology: a critical evaluation. Ultrasound Obstet Gynecol. 2002;19:109-17.

2. Timor-Tritsch IE, Platt LD. Three-dimensional ultrasound experience in obstetrics. Curr Opin Obstet Gynecol. 2002; 14:569-75.

3. Zanforlin Filho SM, Araujo Junior E, Guiaraes Filho HA, Pires CR, Nardozza LM, Moron AF. Sonoembryology by three-dimensional Ultrasonography: pictorial essay. Arch Gynecol Obstet. 2007;276:197-200.

4. Roberts LJ, Bewley S, Mackinson AM, Rodeck CH. First trimester fetal nuchal translucency: problems with screening the general population. Br J Obstet Gynaecol. 1995;102:381-85.

5. Michailidis GD, Papageorgiou P, Economides DL. Assesment of fetal anatomy in the first trimester using two and 3dimensional ultrasound. Br J Radiol. 2002;75(891):215-19.

6. Hirides P, Domali E, Daskalakis G, Papantoniou N, Anastasakis E, Theodora M, et al. 2D or off-line 3D ultrasound during the 1 st trimester scan. Athens University, Athens, Greece. Ultrasound in Obstetrics and Gynecology. 2012;40(1):171-310.

7. Baken L, Rousian M, Koning AH, Bonsel GJ, Steegers E, Exalto N. First trimester detection of structural abnormalities; a comparison of 2D/3D ultrasound and virtual embryoscopy. Ultrasound in Obstetrics and Gynecology. 2012;40(1):171-310.

Cite this article as: Choudhary S, Mishra V, Aggarwal R. Virtual embryoscope for detection of structural abnormalities in first trimester pregnancy. Int J Reprod Contracept Obstet Gynecol. 2017;6:1924-8. 